\title{
Dynamic Arrest in Charged Colloidal Systems Exhibiting Large-Scale Structural Heterogeneities
}

\author{
C. Haro-Pérez, ${ }^{1, *}$ L. F. Rojas-Ochoa,${ }^{2}$ R. Castañeda-Priego,${ }^{3}$ M. Quesada-Pérez,${ }^{4}$ J. Callejas-Fernández, ${ }^{1}$ \\ R. Hidalgo-Álvarez, ${ }^{1}$ and V. Trappe ${ }^{5, \dagger}$ \\ ${ }^{1}$ Grupo de Física de Fluidos y Biocoloides, Departamento de Física Aplicada, Universidad de Granada, 18071 Granada, Spain \\ ${ }^{2}$ Departamento de Física, Cinvestav-IPN, 07360 México Distrito Federal, Mexico \\ ${ }^{3}$ Instituto de Física, Universidad de Guanajuato, 37150 León, Mexico \\ ${ }^{4}$ Departamento de Física, Escuela Politécnica Superior de Linares, Universidad de Jaén, 23700 Linares, Spain \\ ${ }^{5}$ Département de Physique, Université de Fribourg, 1700 Fribourg, Switzerland
}

\begin{abstract}
Suspensions of charged liposomes are found to exhibit typical features of strongly repulsive fluid systems at short length scales, while exhibiting structural heterogeneities at larger length scales that are characteristic of attractive systems. We model the static structure factor of these systems using effective pair interaction potentials composed of a long-range attraction and a shorter range repulsion. Our modeling of the static structure yields conditions for dynamically arrested states at larger volume fractions, which we find to agree with the experimentally observed dynamics.
\end{abstract}

Charged colloids have been extensively used as model systems to study the static and dynamic properties of strongly interacting particles in both in- and out-ofequilibrium states [1]. In many cases their phase behavior could be described by the well established DerjaguinLandau-Verwey-Overbeek theory [2]; a theory that considers the interactions between charged colloids to be determined by repulsive screened Coulomb interactions. More recently, however, experimental and theoretical investigations of highly charged colloidal suspensions showed evidence for the existence of effective long-range attractions between like-charged particles (for a review see [3]). In particular, structural investigations reporting gas-crystal and gas-liquid coexistence associated to the formation of voids [4] led to controversial discussions on the limitation of the Derjaguin-Landau-Verwey-Overbeek theory. Despite the enhanced efforts in understanding the origin of these phenomena, our comprehension of the phase behavior of highly charged colloidal systems remains far from complete. In particular, implications of effective attractions on the dynamical properties of charged colloids remain to be addressed.

In this Letter we report on an experimental investigation of the volume fraction dependent structural and dynamic properties of charged liposome suspensions at quasideionized conditions. For all volume fractions investigated, the suspensions are characterized by structural heterogeneities at large length scales, while exhibiting typical features of strongly repulsive, disordered fluid systems at shorter length scales. We successfully model the static structure factor by effective pair interaction potentials that comprise a long-range attraction and a shorter range repulsion. This modeling reveals an unusual development of the effective particle-particle interactions with volume fraction. In particular, the position of the repulsive barrier ceases to de- crease at large volume fractions, which causes a dynamic arrest of the system. Our findings indicate that in highly charged colloidal systems the formation of structural heterogeneities and the dynamic arrest have a common origin.

Our liposomes are composed of phosphatidylserine (PS) and egg phosphatidylcholine $(\mathrm{PC})$ at a ratio of $\mathrm{PS} / \mathrm{PC} \sim 1$. They are prepared by using the technique described in Ref. [5], yielding unilamellar vesicles with a mean diameter of $d=120 \pm 12 \mathrm{~nm}$ as determined by static and dynamic light scattering. For the $p \mathrm{H}$ conditions used in this study $(p \mathrm{H} \sim 7)$ the zwitterionic phospholipid PC is uncharged, such that the total surface charge of the liposome is determined by the monovalent ionic phospholipid PS. We estimate the number of dissociated charges to be $\sim 2000$ electrons per particle. Because of the small size and high surface charge, shape fluctuations are insignificant, such that our vesicles can be considered as charged spherical colloids. We prepare suspensions with volume fractions ranging from $\phi=0.009$ to $\phi=0.12$. To ensure the lowest possible ionic strength, we seal the suspensions in contact with a mixed bed of ion exchanger resin in cylindrical quartz cells 5 days prior to our light scattering experiments. Such treatment with an ionic exchange resin results for pure water in a reduction of the ionic strength from $10^{-5}$ to $10^{-6} M$. None of our samples exhibit the typical iridescence, which is usually observed when charged colloids organize into crystals at low ionic strength. Instead, for $\phi$ up to 0.06 we observe that the viscosity gradually increases during a few hours after the liposome dispersion has been put into contact with the resin. For the two largest $\phi$ investigated, $\phi=0.09$ and $\phi=0.12$, we find a strikingly different behavior. Shaking up the just prepared mixture of dispersion and resin transforms the sample into an elastic solid that withstands even rigorous shaking. Upon addition of a small quantity of a 
concentrated electrolyte solution, the system reverts back to a fluid state that again transforms into a solid as the ions are removed by the resin.

To explore the origin of this remarkable behavior we investigate the structure and dynamics of our dispersions by static light scattering and dynamic light scattering using a commercial light scattering device. Indeed, our systems fulfill nearly optimal conditions for light scattering, as we expect multiple scattering to be negligible, since the refractive index of our liposomes is 1.36, close to that of water 1.33. To ensure a proper ensemble average of the light scattered by our solidlike samples, we rotate these samples during the measurements [6].

From the ensemble-averaged intensity $I(q)$, we determine the static structure factor $S(q) \approx\left(\phi_{0} / \phi\right) \times$ $\left[I(q) / I_{0}(q)\right]$, where $I_{0}(q)$ is the light intensity scattered by a dilute suspension of noninteracting particles of volume fraction $\phi_{0}$. For the samples with $\phi$ ranging from 0.009 to 0.06 the structure factor exhibits a pronounced particle-particle peak, whose position $q_{\max }$ strongly depends on $\phi$, as shown in Fig. 1(a). As typically found for strongly repulsive systems, the mean interparticle distance, $r_{m} \approx 2 \pi / q_{\max }$, scales as $\phi^{-1 / 3}$, as shown in Fig. 1(b). Unfortunately, though we clearly observe an increase of $S(q)$ at large $q$, for the solidlike samples $(\phi=0.09$ and $\phi=0.12)$ the peak positions are shifted to $q$ values that
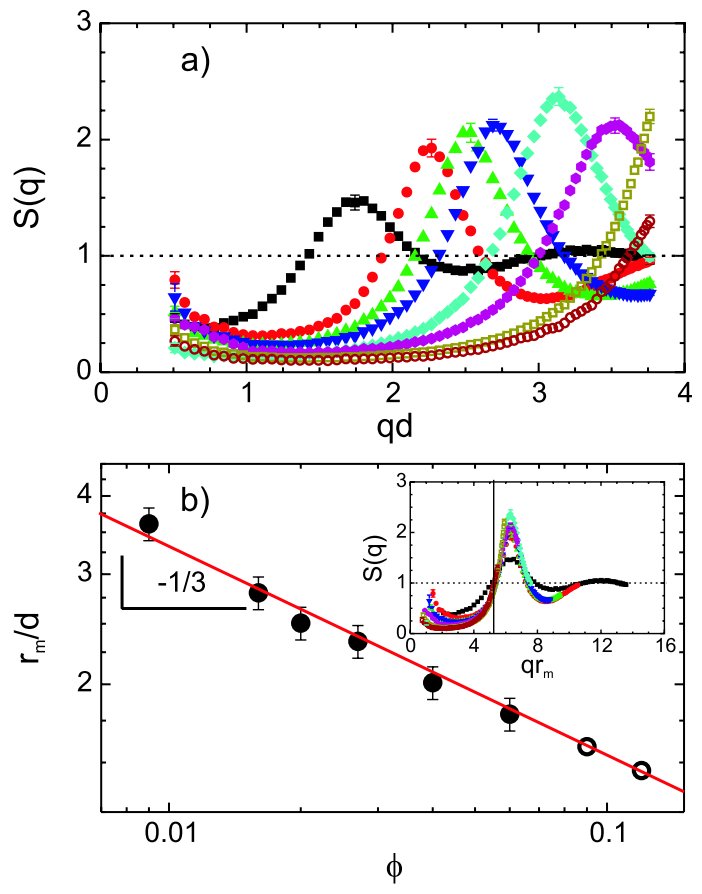

FIG. 1 (color online). (a) $q$ dependence of $S(q)$ : from left to right, $\phi=0.009,0.016,0.020,0.027,0.040,0.060,0.09$ 0, 0.120 . (b) $r_{m}$ versus $\phi$. Solid circles, data obtained from $q_{\max }$; open circles, $r_{m}$ for $\phi=0.09$ and 0.120 as expected from extrapolation; solid line, power-law fit to the data. Inset: $S(q)$ as a function of $q r_{m}$. The vertical line indicates the $q$ value where $S(q) \approx 1, q$ value at which we determine the intensity correlation function. are beyond the $q$ range accessible in our experiments. To test whether the peak position of these systems follows the same trend as the one observed at lower $\phi$, we use the $r_{m}$ values obtained by extrapolation (open circles in Fig. 1(b)) to normalize the $q$ axis. As shown in the inset of Fig. 1(b), our scaling procedure leads to a reasonable coincidence of all $S(q)$ data around the peak, which indicates that the monotonic decrease of $r_{m}$ with $\phi^{-1 / 3}$ persists for the two largest volume fractions investigated. This scaling in principle denotes that the particles configure to achieve a maximal distance between them, which indirectly implies that space is homogeneously filled with particles. In seeming contradiction to this, we find for all $\phi$ investigated that $S(q)$ exhibits an upturn at low $q$, indicative of large-lengthscale heterogeneities in the particle distribution [7].

This low $q$ rise in $S(q)$ is reminiscent of attractive colloidal systems, where aggregation of the particles naturally leads to the formation of large-length-scale structures. However, in a short-range attractive system the particles touch each other and generally exhibit a mean particle distance that is independent of volume fraction. To account for the behavior observed here we consider effective pair potentials, $U(r)$, comprising a repulsive and a long-range attractive component. The specific shape of the potentials is obtained by approximating the experimental $S(q)$ by numerical solutions of the Ornstein-Zernike equation, where we use the soft mean spherical approximation as closure relation [8]. We partially account for polydispersity taking the differences in the optical properties of different sized particles into account, but assuming the same interactions between them; the size distribution is approximated by a discrete Schulz distribution composed of three different sized liposomes [9]. For the two most concentrated samples, for which we do not access the particle-particle peak experimentally, we constrain the parameters to also reproduce the peak position as determined by extrapolation [see Fig. 1(b)]. The potentials best reproducing the experimental $S(q)$ data are shown in Fig. 2(a). To test whether these potentials indeed lead to a particle configuration that agrees with the experimental $S(q)$, we perform Monte Carlo simulations at constant volume (MC-NVT), using $N=5832$ particles in a cubic box of volume $L^{3}=$ $N / n$ and periodic boundary conditions in each direction, where $n$ is the number density. The structure factors obtained from these simulations are in good agreement with the experimental ones, as shown for $\phi=0.009,0.027$, and 0.09 in Figs. 2(b)-2(d), respectively. This agreement corroborates the validity of our effective particle interaction potentials. Moreover, by contrast to the voids that have been previously observed in highly charged systems [4], our simulations reveal that the structural heterogeneities are in our systems not well defined. Indeed, the direct comparison of the equilibrium configurations of our systems with equivalent repulsive systems reveals only little differences, while an analysis of the local particle density 

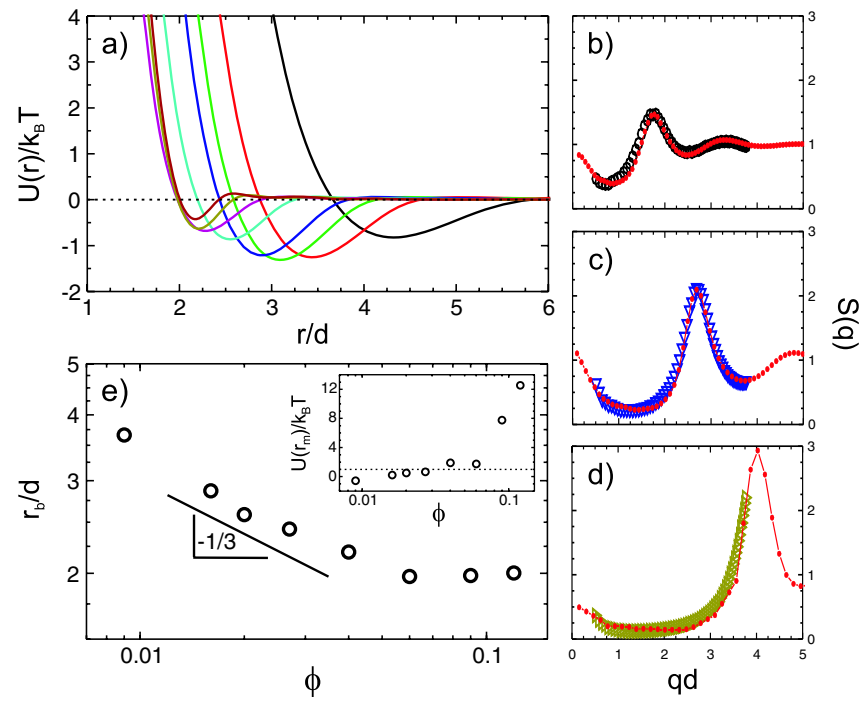

FIG. 2 (color online). (a) Effective interparticle energy estimated from $S(q)$ and used in MC-NVT simulations: from right to left $\phi=0.009,0.016,0.020,0.027,0.040,0.060,0.090,0.120$. Comparison of $S(q)$ obtained from simulation (solid symbols) and experiment (open symbols) for (b) $\phi=0.009$, (c) $\phi=0.027$, and (d) $\phi=0.090$. (e) $\phi$ dependence of $r_{b}$. Inset: effective interaction energy at $r_{m}$ as a function of $\phi$. Dashed line: $U\left(r_{m}\right) / k_{B} T=1$.

clearly exposes spatial heterogeneities in the particle distribution, as shown in [7].

The effective interaction potentials of our liposomes exhibit a remarkable $\phi$ dependence, as shown in Fig. 2(a). The depth of the attractive minimum becomes deeper as $\phi$ increases, reaches a maximal depth at $\phi=$ 0.02 , and then becomes again less pronounced as $\phi$ is further increased. More intriguingly, the position of the repulsive barrier systematically shifts to smaller distances with increasing $\phi$ in the low volume fraction range to then remain approximately constant for $\phi \geq 0.06$, seemingly indicating that the increase in the counterion concentration with increasing $\phi$ does not lead to further screening of the surface charge in the high volume fraction range. To capture this feature we determine the onset of the repulsive behavior $r_{b} / d$ where $U(r)=0$, reporting the result in Fig. 2(e). A comparison with the $r_{m} / d$ data shown in Fig. 1(b) reveals that $r_{m} \approx r_{b}$ for $\phi \leq 0.06$ while $r_{m}$ becomes significantly smaller than $r_{b}$ for $\phi=0.09$ and $\phi=0.12$. This indicates that for the two solidlike samples the particles are squeezed together to sense the strong repulsion between them. This is most clearly seen in the inset of Fig. 2(e), where we report the magnitude of the interparticle energy at $r_{m}$. While $U\left(r_{m}\right)$ varies insignificantly around $1 k_{B} T$ for the fluidlike samples, $\phi \leq 0.06$, it increases to $8 k_{B} T$ and $13 k_{B} T$ for $\phi=0.09$ and $\phi=0.12$, respectively.

To test whether the $\phi$ dependence of $U\left(r_{m}\right)$ reflects on the dynamical properties of our systems, we measure the intensity autocorrelation function, $g_{2}(q, t)$, at $q r_{m} \approx 5$, indicated by the vertical line in the inset of Fig. 1(b). At this $q r_{m}$ value $S\left(q r_{m}\right) \sim 1$, such that $g_{2}(q, t)$ is mainly determined by the self-motion of the particles [10]. For the fluidlike systems we use the Siegert relation $g_{2}(q, t)=$ $1+g_{1}^{2}(q, t)$ to convert $g_{2}(q, t)$ in the electric field autocorrelation function, $g_{1}(q, t)$. For the solidlike systems, we determine $g_{1}(q, t)$ using the ensemble-averaging procedure described in Ref. [6]. Our dynamic light scattering data confirm the occurrence of a transition from a fluidlike state at $\phi \leq 0.06$ to a solidlike state at $\phi \geq 0.09$. As shown in Fig. 3(a), $g_{1}(q, t)$ decays completely for $\phi \leq 0.06$, while $g_{1}(q, t)$ is characterized by a nondecaying component at long times for $\phi=0.09$ and $\phi=0.12$, revealing the dynamically arrested state of these systems. The dynamics of all our fluidlike systems is characterized by a two-step decay. This indicates that the particle dynamics is constrained by cages formed by the surrounding particles at all volume fractions investigated. While this constraint is eventually released by the structural rearrangement of the
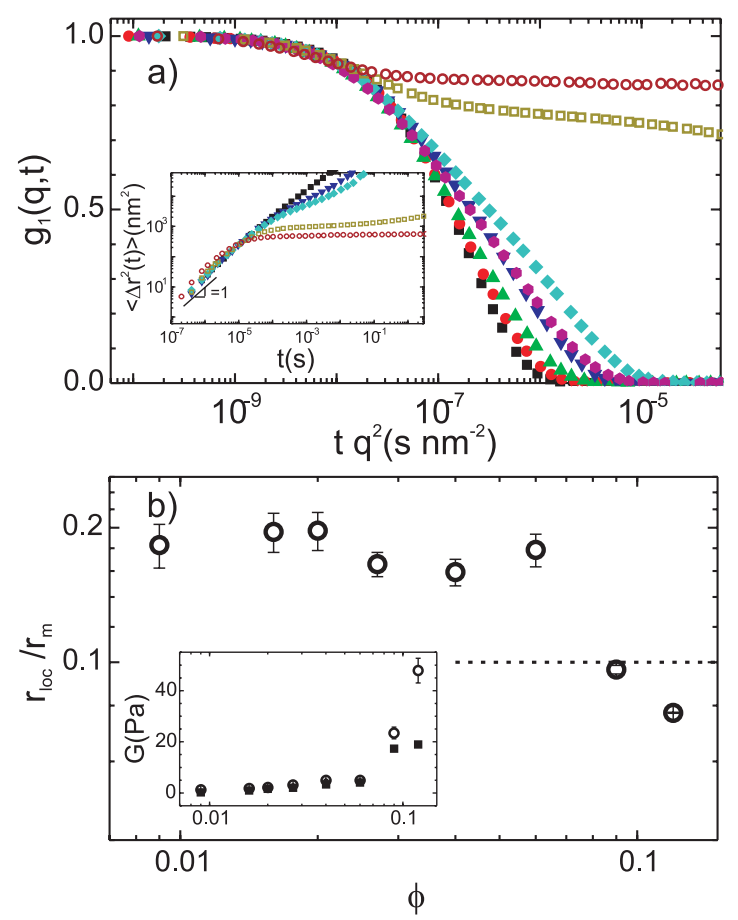

FIG. 3 (color online). (a) $g_{1}(q, t)$ determined at $q \approx 5 / r_{m}$ for $\phi=0.009$ (black, solid squares), 0.016 (red, solid circles), 0.020 (green, solid triangle up), 0.027 (blue, solid triangle down), 0.040 (light blue, solid diamond), 0.060 (magenta, solid hexagon), 0.090 (dark yellow, open squares), 0.120 (wine, open circles). To account for the change in $q$ at which we measure $g_{1}(q, t)$, the time axis is normalized by $q^{2}$. Inset: time dependence of $\left\langle\Delta r^{2}(t)\right\rangle$ for $\phi=0.009,0.027,0.040,0.090$, and 0.120. (b) $\phi$ dependence of $r_{\text {loc }}$ in units of $r_{m}$. Dashed line corresponds to the Lindemann criterion, $r_{\mathrm{loc}} / r_{m}=0.1$. Inset: comparison of the elastic modulus as estimated using the microrheology approach $\left(G_{d}\right.$, open symbols) and as calculated from the interparticle potential $\left(G_{s}\right.$, solid symbols). 
cage in the case of the fluidlike samples, it is permanent in the case of solidlike samples.

To quantify the constraints set by the cage we convert $g_{1}(q, t)$ into mean squared displacement $\left\langle\Delta r^{2}(t)\right\rangle$ according to $g_{1}(q, t)=\exp \left[-\frac{1}{2} q^{2}\left\langle\Delta r^{2}(t)\right\rangle / 3\right]$. The results are shown in the inset of Fig. 3(a), where we omit for clarity the data obtained for $\phi=0.016,0.02$, and 0.06 . We characterize the maximal displacement of the particles within the cage by a localization length, $r_{\mathrm{loc}}=\left[\left\langle\Delta r^{2}(t)\right\rangle / 3\right]^{1 / 2}[11]$, which we determine from $\left\langle\Delta r^{2}(t)\right\rangle$ using a scaling method [12]. To evaluate the arrest conditions in terms of the Lindemann criterion we normalize $r_{\text {loc }}$ with $r_{m}$ [13]; this criterion demands that $r_{\text {loc }} / r_{m} \approx 0.1$ at the liquid-solid transition [14]. Consistent with a transition between $\phi=0.06$ and $\phi=0.09$, we find that $r_{\text {loc }} / r_{m}$ is $\sim 0.2$ for all fluidlike samples, while it abruptly drops to values below 0.1 for $\phi \geq 0.09$, as shown in Fig. 3(b). This behavior is strongly reminiscent of the abrupt change observed in $U\left(r_{m}\right)$ and suggests that the transition to dynamically arrested states is correlated to the peculiar development of the repulsive barrier.

To explore this correlation, we convert both $r_{\text {loc }}$ and $U(r)$ into elastic moduli, $G$, which we use as a measure of the energy content of our systems. For $r_{\text {loc }}$ we use the microrheology approach, $G_{d}=k_{B} T /\left[r_{m}\left(3 r_{\text {loc }}^{2}\right)\right]$ [15]. For $U(r)$ we use the Zwanzig and Mountain model $G_{s}=n k_{B} T+$ $\frac{2 \pi}{15} n^{2} \int_{0}^{\infty} g(r) \frac{d}{d r}\left(r^{4} \frac{d U(r)}{d r}\right) d r$ [16]. Both approaches yield elastic moduli that compare remarkably well, as shown in the inset of Fig. 3(b). While $G$ varies insignificantly around $2 \mathrm{~Pa}$ for $\phi \leq 0.06$, it significantly increases for the two solidlike samples. Though the agreement between $G_{d}$ and $G_{s}$ is less convincing at the largest volume fraction, the basic feature of the transition is captured in both measures. This result is remarkable as it demonstrates that we can use the static data to predict the dynamics of our system.

Note that our approach is an entirely empirical one. We do not a priori assume a specific model, but simply use the static structure factor to obtain a gauge of an effective pair potential. The peculiar development of this potential with volume fraction indicates that the dynamical arrest is in our highly charged system not just due to an overcrowding effect, as this is the case for hard-sphere colloids at the glass transition; it appears to be due to the arrest of the repulsive barrier. As we do not expect such arrest for equivalent purely repulsive systems [17], the development of the repulsive barrier must be directly linked to the low $q$ rise in $S(q)$, indicative of effective attractions. Thus, our results effectively indicate that the formation of structural heterogeneities and the dynamic arrest have a common origin. To our knowledge our findings are the first disclosing this relation; this implies that any model describing the appearance of cohesive energy in highly charged colloidal systems will need to account for the peculiar development of the repulsive barrier as well. We could envision that the uneven distribution of counterions inherent to the heterogeneous distribution of the particles is at the origin of both effective attraction and arrest of the repulsive barrier.

We thank the ERDF, the I + D + I project MAT200612918-C05-01 -02 (C. H. P., J. C. F., M. Q. P., R. H. A), the Swiss National Science Foundation (L. F. R. O. and V. T.), and Conacyt-Mexico Grants No. 51669 and No. 46373 (L.F. R. O. and R. C.P.) for financial support. We are indebted to J. Estelrich for providing us with the liposome dispersions investigated and to P. Schurtenberger for the opportunity to use the infrastructure of his laboratory.

*Present address: Instituto de Física "Manuel Sandoval Vallarta," UASLP, 78000 San Luis Potosí, Mexico.

†Veronique.Trappe@unifr.ch

[1] A. Yethiraj, Soft Matter 3, 1099 (2007).

[2] B. V. Derjaguin and L. Landau, Acta Physicochim. (URSS) 14, 633 (1941); E. J. Verwey and J. T.G. Overbeek, Theory of the Stability of Lyophobic Colloids (Elsevier, Amsterdam, 1948).

[3] B. V. R. Tata and S. S. Jena, Solid State Commun. 139, 562 (2006).

[4] K. Ito, H. Yoshida, and N. Ise, Science 263, 66 (1994); H. Yoshida, N. Ise, and T. Hashimoto, J. Chem. Phys. 103, 10146 (1995); B. V. R. Tata et al., Phys. Rev. Lett. 78, 2660 (1997).

[5] C. Haro-Pérez et al., Colloids Surf. A 270, 352 (2005).

[6] P. N. Pusey and W. van Megen, Physica A (Amsterdam) 157, 705 (1989); W. van Megen, S. M. Underwood, and P. N. Pusey, Phys. Rev. Lett. 67, 1586 (1991).

[7] See EPAPS Document No. E-PRLTAO-102-071901 for supplementary material. For more information on EPAPS, see http://www.aip.org/pubservs/epaps.html.

[8] G. Zerah and J. P. Hansen, J. Chem. Phys. 84, 2336 (1986).

[9] G. Nägele, Phys. Rep. 272, 215 (1996).

[10] P. N. Pusey, J. Phys. A: Math. Gen. 11, 119 (1978).

[11] S. D. Wilke and J. Bosse, Phys. Rev. E 59, 1968 (1999).

[12] The $\left\langle\Delta r^{2}(t)\right\rangle$ data obtained at different $\phi$ are shifted onto the one of $\phi=0.12$, to obtain the best overlap in the fast dynamics and transition regime. The factors used to shift $\left\langle\Delta r^{2}(t)\right\rangle$ are then used to determine $r_{\text {loc }}$ relative to the one obtained for $\phi=0.12$, where $r_{\text {loc }}$ is clearly defined as the long-time limit of $\left\langle\Delta r^{2}(t)\right\rangle$.

[13] X. Xia and P. G. Wolynes, Proc. Natl. Acad. Sci. U.S.A. 97, 2990 (2000).

[14] F. A. Lindemann, Physikalische Zeitschrift 11, 609 (1910).

[15] T. G. Mason, Hu Gang, and D. A. Weitz, J. Opt. Soc. Am. A 14, 139 (1997).

[16] R. Zwanzig and R. D. Mountain, J. Chem. Phys. 43, 4464 (1965).

[17] Calculations of the potentials of a purely repulsive system, with parameters chosen to reproduce the peak height in $S(q)$ of our liposome system, show that for a purely repulsive system the screening length should continuously decrease with increasing $\phi$ in the $\phi$ range investigated [9]. 